\title{
A Differential Reflection-Type Phase Shifter Based on CPW Coupled- Line Coupler in 45nm CMOS SOI
}

\author{
R. Azhar Shaheen ${ }^{\# 1}$, Rehman Akbar", Timo Rahkonen ${ }^{\$}$, Janne Aikio ${ }^{\$}$, Alok Sethi ${ }^{\#}$, Aarno Pärssinen ${ }^{\#}$ \\ ${ }^{\#}$ Center for Wireless Communication, University of Oulu, Finland \\ ${ }^{\$}$ Circuits and Systems, University of Oulu, Finland \\ ${ }^{1}$ rana.shaheen@oulu.fi
}

\begin{abstract}
This paper presents a mm-wave fully differential coplanar waveguide (CPW) vertically-coupled lines coupler and a reflection type phase shifter (RTPS) based on the proposed coupler. The use of grounded strip line structure for vertical coupling in CPW based coupler provides not only the improved coupling properties than the edge coupling but also provides the opportunity of using surrounding area efficiently to reduce area. The proposed coupler and phase shifter are designed and fabricated in 45nm CMOS SOI technology. Measured insertion loss of the coupler is $\sim 6 \mathrm{~dB}$ from 18 to $43 \mathrm{GHz}$. A digitally controlled parallel LC load is used for controlling the phase of the phase shifter. Measured phase tuning range is $\mathbf{1 1 5}$ degrees, with insertion loss variation of 4.5 to $9 \mathrm{~dB}$, for a frequency range of 39 GHz to $45 \mathrm{GHz}$. Total drawn area of the phase shifter is 0.214 $\mathrm{mm}^{2}$. The area of the proposed RTPS is smaller than the recently published state-of-the-art structures.
\end{abstract}

Keywords- Phase shifter, Hybrid Coupler, Phased Array, 5G, mmWave, CMOS SOI.

\section{INTRODUCTION}

Recent developments in different types of communication media systems, such as, video on demand, internet of things, and live streaming, etc. requires a clear demand in bandwidth of the channel. Due to the availability of wider bandwidths, mmWave frequencies, such as, $24 \mathrm{GHz}, 28 \mathrm{GHz}$ and $38 \mathrm{GHz}$. are considered as the potential operating bands for $5 \mathrm{G}$ communication systems. In addition to beam steering capability of phased arrays, they are also used to increase the combined gain of the antenna aperture, made up of multiple small antenna elements. CMOS or BiCMOS technologies provide an opportunity to fully integrate larger phased array systems. CMOS phase shifters are key building blocks for such phased array systems. Depending on the system requirements, passive (e.g. RTPS) or vector modulator (VM) based active phase shifters are being used for example in [2] - [5]. Differential phase shifters offer not only the benefit of covering double the amount of phase shift by inverting the signals, but also less prone to inter-channel coupling between the phased array elements.

CMOS RTPSs can benefit from their compact size and high-resolution phase shifting properties at the expense of higher IL variation. RTPS is mainly composed of two building blocks, i.e. quadrature coupler and reflective load. Quadrature couplers are implemented with the help of lumped-elements [2] or transmission line (TL) based structures [1], [3]-[5]. TL-based couplers are implemented by using either the vertical or edge coupling between two coupled lines. Vertical or lateral coupling is useful for differential implementation of the coupler design [1] [2] [3]. Differential RTPS proposed in [3] and [4] are based on vertically-coupled strip lines couplers, however, they consume larger chip area. Grounded microstrip line-based couplers offer the opportunity to use the surrounding area efficiently with minimum effect of unwanted coupling from surrounding structures of the RTPS, i.e. reflective load. A CPW based coupler is presented in [5], however, the structure is single-ended.

To address the above-mentioned area constraints of the couplers in RTPS design, we propose a fully differential CPWbased vertically-coupled line coupler and an RTPS operating at $40 \mathrm{GHz}$ centre frequency based on the proposed coupler. Because of the shielding of signal lines, CPW-based coupler can be implemented in a meander-line configuration for compact design. The proposed coupler and an RTPS are fabricated in 45nm CMOS SOI technology. Designed coupler and RTPS structures are bidirectional and can be used in receivers and transmitters. The proposed RTPS is smaller in area than the recently published state-of-the-art works [3] [4]. Section II describes the design of the proposed coupler and the reflection load. Section III shows the simulated and measured
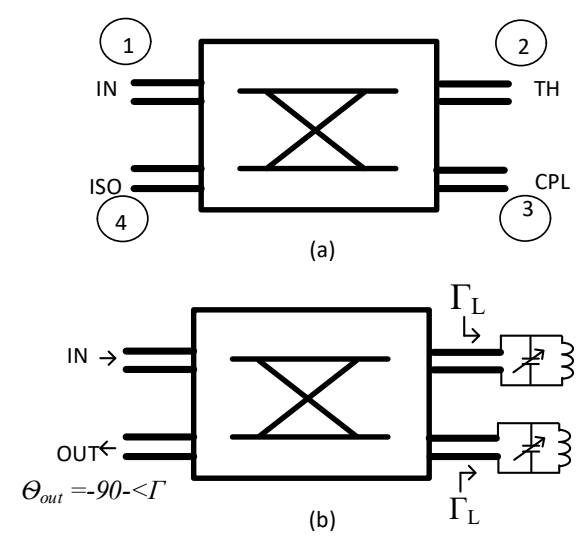

Fig 1. (a) Coupler with port definition, (b) Reflection-type phase shifter block diagram

results of the coupler and RTPS. Conclusions are given in section IV.

\section{Circuit Design}

A quadrature coupler is a four-port device, as shown in Fig. 1(a). Input signal is applied at port 1 and splitted into two signals at through and coupled ports. A reflection-type phase shifter is composed of a $90^{\circ}$ coupler and a reflection load. A 
block diagram of a differential reflection-type phase shifter is shown in Fig. 1 (b). Input signal is applied at port 1, and a phase shifted version is taken out at isolation port, i.e. port 4, as shown in Fig. 1 (b). Identical reflection loads are connected at the through (port2) and at the coupled port (port3). From these ports, input signal is reflected back and combined at isolation port with additional phase shift, equivalent to the angle of the reflection coefficient of the reflection loads. Upon changing the reflection coefficient, a change in the phase shift can be seen at the output. The amount of phase tuning range of an RTPS depends on the passive load design. In this work, we use a parallel connection LC load to achieve a phase tuning range of 115 degrees by using capacitive tuning.

\section{A. Coupler design using vertically-coupled lines}

A 90 degree coupler can be implemented in multiple ways, e.g. transformer-based lumped element couplers [2] and coplanar strip line (CPS) couplers [3]. CPS based couplers offer wideband operation and lower load variation sensitivity [3]. Coupled line structures can be characterized by using even and odd mode impedance analysis. Higher the difference between the even mode and odd mode impedances, better is the coupling between the lines. Vertical coupling provides benefits of high coupling ratio as well as good input and output matching conditions [1]. In even mode excitation in vertically coupled lines, inductance in the line is increased and capacitance is decreased because of same polarity current flowing in both lines. As a result, even mode impedance is increased. Similarly, in odd mode excitation, inductance of the line is decreased and capacitance is increased because of opposite polarity current is flowing through the lines. On the other hand, edge-coupled line couplers are easy to implement for single-ended structures, but they get more complicated for differential structures. Due to the higher coupling and symmetric nature of vertically-coupled lines, they are suitable candidates to be used for designing differential couplers. In [1], [3] and [4], vertical-coupled couplers are presented. To minimize high conductive substrate losses, floating metal stripes are used below the structures which results in asymmetry between the top and bottom conductors. In [4], to compensate this effect, an asymmetrical width of top and bottom conductors was used to meet the requirements of even and odd mode impedances. A symmetrical and diagonal configuration was used in [3] to balance the asymmetry.

Even and odd mode impedances $\left(\mathrm{Z}_{\mathrm{o}, \mathrm{e}}\right.$ and $\mathrm{Z}_{\mathrm{o}, \mathrm{o}}$, respectively) of coupled lines are given by (1) and (2).

$$
\begin{aligned}
& Z_{o, e}=Z_{o} \sqrt{\frac{1+c}{1-c}} \\
& Z_{o, o}=Z_{o} \sqrt{\frac{1-c}{1+c}}
\end{aligned}
$$

where $\mathrm{c}$ is coupling ratio between the lines, from 0 to 1 . For $-3 \mathrm{~dB}$ coupling, i.e. $\mathrm{c}=0.7$, and reference characteristic impedance of $75 \mathrm{Ohms}, \mathrm{Z}_{\mathrm{oe}}$ and $\mathrm{Z}_{\mathrm{oo}}$ are 178 and $31 \mathrm{Ohms}$, respectively.
In this work, we designed a vertically-coupled coupler using $45 \mathrm{~nm}$ CMOS SOI process technology, which provides great benefit of high resistive substrate, hence, provides better performing passives structures. Additionally, availability of two ultra-thick metals in its back-end-of-the-line (BEOL) metal stack, allows the design of vertically-coupled coupler with good coupling (i.e. $-3 \mathrm{~dB}$ ), symmetrical structure and good matching conditions. Differential coplanar waveguide (CPW) lines are utilized. Ground line shielding in CPW is used to minimize the impact of dummy structures below and surroundings. Secondly, utilizing the chip area efficiently by the meander line method. CPW configuration not only provides benefit of shielding the lines from surroundings and thus reduce unwanted coupling but also provides additional freedom for design parameters such as, spacing, clearance, width, etc. to design characteristic impedances of the lines. The final structure is simulated with electromagnetic (EM) tool (ADS momentum). Simulated values of even and odd-mode impedances are 175 and $27 \mathrm{Ohms}$, respectively, which are close to the specification.

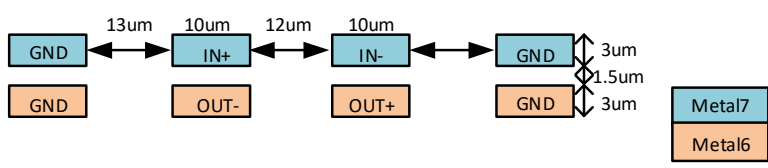

Substrate

Fig 2. Cross-section of vertically coupled CPW lines

\section{B. Tunable Reflective Load}

Tuning range of a reflection type phase shifter depends on the tuning range of the reactance $\mathrm{X}_{\mathrm{L}}$ of the identical loads at through and coupled ports. Either one of the elements can be tuned to perform the fine step change in $\mathrm{X}_{\mathrm{L}}$. Generally, due to the lower complexity and higher tuning range, capacitance of the load is tuned. Inductor tuning can also be used but it brings more losses with low tuning resolution. However, a combination of inductive and capacitance tuning can be possible. Tuning range of the reactance increases with increased number of tunable elements, at the cost of additional loss. There are different types of loads which can be used to change the reactance of the load, e.g. series LC resonant load, parallel LC resonant load, CLC $\pi$-resonant load and transformer based multi-resonant based load [2]. Depending on the required tuning range of the phase shifter, either one of the above load types can be selected.

In this work, we used capacitance tuning for tuning the reactance of a parallel LC load. Capacitance tuning can be implemented by using switched capacitors or varactors. Typical tuning ratio (TR) of varactors is $\sim 4$ [2]-[4], while switched capacitor circuits can achieve higher TR, e.g. $\sim 10$. Switch capacitors are based on nmos or pmos switches, which brings additional design constraints at mmWave frequencies, i.e. onstate resistance $\left(\mathrm{r}_{\mathrm{on}}\right)$ and off-state capacitance $\left(\mathrm{C}_{\mathrm{off}}\right)$. Finite series $r_{\text {on }}$ of the switch in $\mathrm{ON}$-state degrades the quality factor of the capacitance, while $\mathrm{C}_{\text {off }}$ of the switch in off-state limits the TR. Therefore, at mmWave frequency designs, there is always 
a tradeoff between $\mathrm{R}_{\text {on }}$ and $\mathrm{C}_{\text {off }}$ of the switch when optimizing the quality factor of the tank and TR.

Capacitor bank is composed of four single cells, as shown in Fig 3 (a), with minimum capacitance value of $30 \mathrm{fF}$ and maximum capacitance value of $60 \mathrm{fF}$ with $10 \mathrm{fF}$ step. Capacitance value seen by the load is $30 \mathrm{fF}$ when only switches $\mathrm{M} 1_{\mathrm{a}, \mathrm{b}}$ are turned $\mathrm{ON}$, while maximum capacitance $(180 \mathrm{fF})$ is seen by the load when all the switches (M1 to M4) are turned ON, providing $\mathrm{TR}=6$. Metal insulator metal (MIM) capacitors with high quality factor are used in differential termination. Inductor is designed using ultra thick metal 6 of BEOL as shown in Fig 3 (b). Simulated inductance and quality factor are shown in Fig 3 (c). Tuning range of reflection coefficient of the parallel LC load is shown in Fig 3 (d). A tuning range of more than $150^{\circ}$ is achieved by sweeping the whole control range of the capacitances.

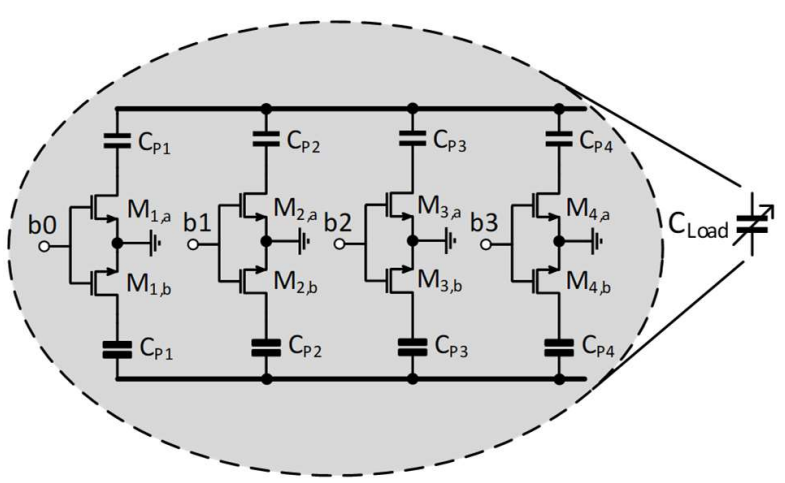

(a)
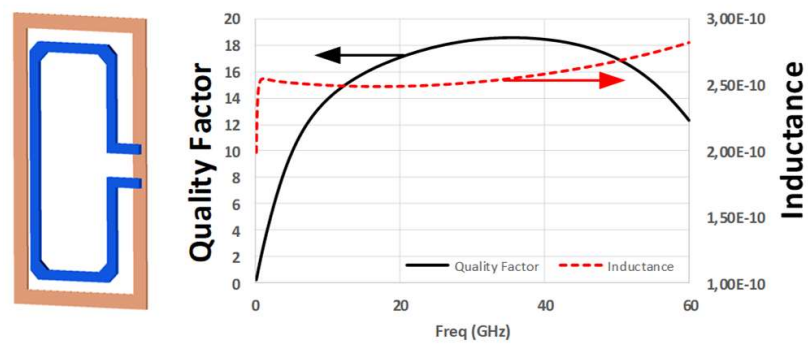

(b)

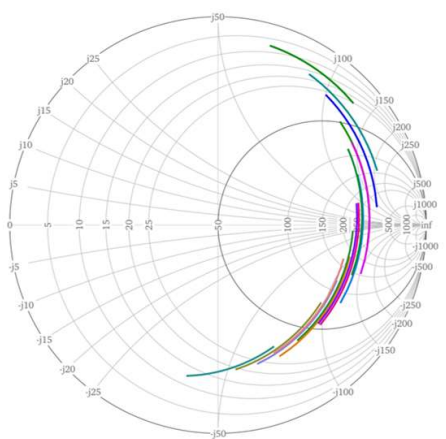

(c)

Fig 3. (a) Digitally tuneable switch capacitors, (b) 3D layout,for load inductor and EM simulated quality factor and inductance of load inductor, (c) Simulated curves of S11 of LC load when capacitance value is varied

\section{RESULTS}

A differential coupler as a separate structure and a reflection-type phase shifter are fabricated using 45nm CMOS SOI process. Due to the complexity of differential measurement setup, differential coupler is designed in coupled configuration, i.e. only input and coupled ports can be measured, while other ports are terminated with $100 \mathrm{Ohm}$ on-chip resistors, shown in Fig 4. Die micrograph of RTPS is shown in Fig 5, and it draws an area of 595 um x 357 um. The coupler is drawn in an Sshaped form such a way that the empty area in this structure is filled by reflective load of the phase shifter. Differential probes are used to measure the structures with Keysight $67 \mathrm{GHz}$ PNA. Calibration up to the probe tips is performed from frequencies $200 \mathrm{MHz}$ to $52 \mathrm{GHz}$. $3 \mathrm{~dB}$ coupled measurements show an insertion loss of $5.5 \mathrm{~dB}$ to $6 \mathrm{~dB}$ from $18 \mathrm{GHz}$ to $43 \mathrm{GHz}$. Measured and simulated responses are given in Fig 6. Measured amplitude and phase response for all possible switching states of RTPS is shown in Fig 7 and Fig 8, respectively. Phase shift range of RTPS is $115^{\circ}$, and $6.5 \mathrm{~dB} \pm 2 \mathrm{~dB}$ loss variation is achieved from 39 to $45 \mathrm{GHz}$ frequency range. Digital control of capacitance switches of the reflective load is implemented with the help of on-chip shift registers. A higher phase shifting range, e.g. $140^{\circ}$ can be achieved at the cost of higher IL variation. These measurements include the loss of pads and additional transmission line extensions.

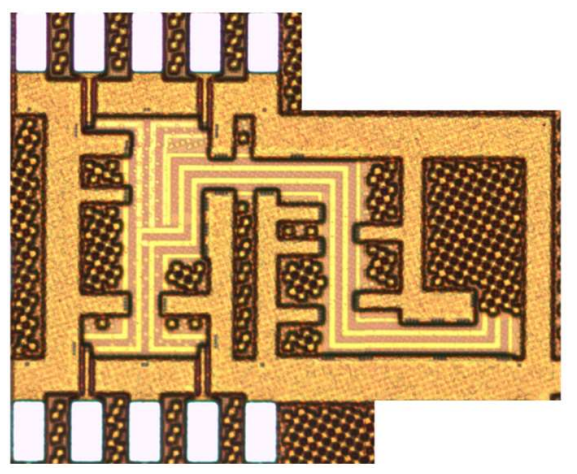

Fig 4. Die micrograph of the coupler

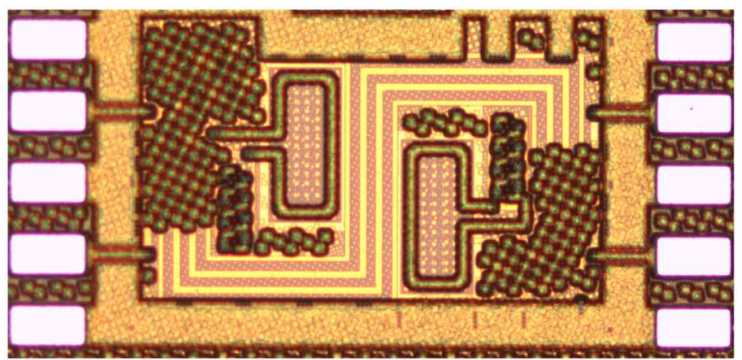

Fig 5. Die micrograph of the reflection-type phase shifter. 


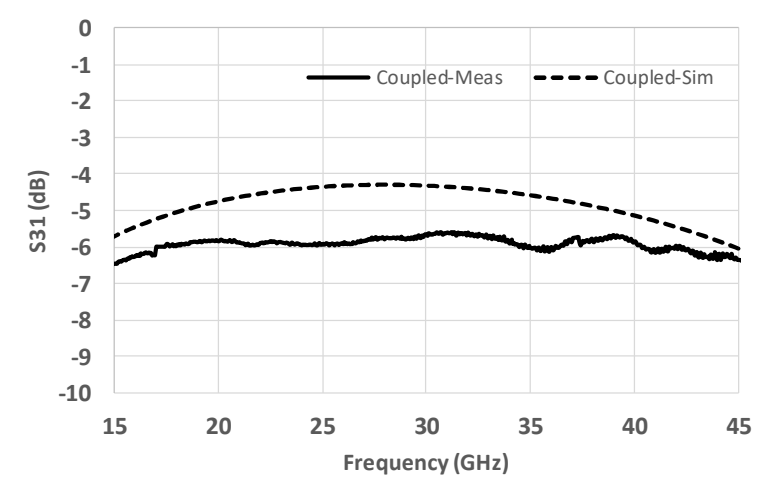

Fig 6. Measured and EM-simulated response of coupled port of proposed coupler. Measured result includes the losses of pads and additional transmission lines.

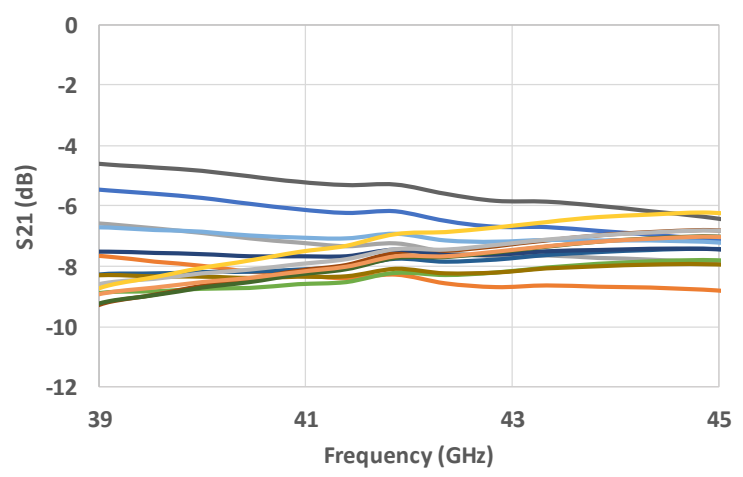

Fig 7. Measured amplitude response for different load tuning states

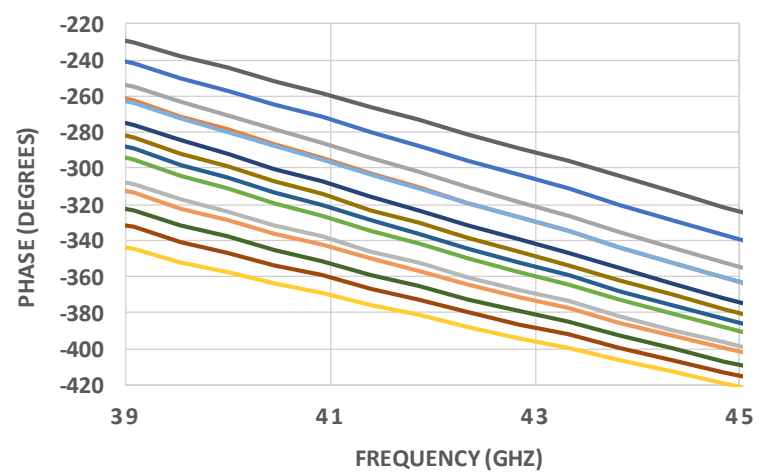

Fig 8. Measured phase responses for different load tuning states

\section{CONCLUSION}

A compact $90^{\circ}$ fully integrated differential coupler based on coplanar waveguide (CPW) lines is presented for mmWave frequency systems. Designed coupler is based on symmetrical vertical coupling between the shielded differential lines. Measured results of coupled port show an insertion loss of $6 \mathrm{~dB}$ from $18 \mathrm{GHz}$ to $43 \mathrm{GHz}$ frequencies when losses of the pads and additional transmission lines are not excluded. A reflection type phase shifter is also designed and presented based on the proposed coupler and switch capacitor as tuneable reflection load. Measured phase tuning range of the phase shifter is $115^{\circ}$, with insertion loss of $6 \mathrm{~dB} \pm 2 \mathrm{~dB}$. Use of vertical coupling and differential $\mathrm{CPW}$ configuration provides an opportunity to utilize the area efficiently without the impact of parasitic coupling from surrounding structures. High variation in IL of RTPS is mainly due to the response of switchable capacitance due to lower Q-value, which can be mitigated by using varactors at the cost of lower TR. Nevertheless, implementing the varactors will not eliminate the advantage of reduced area of the structure. Coupler and RTPS structures are designed and fabricated using $45 \mathrm{~nm}$ CMOS SOI technology. Total drawn area of the RTPS is $0.214 \mathrm{~mm}^{2}$ without pads. With respect of area, the differential CPW coupler based on RTPS in this work outperforms the other state-of-the-art design [3] as shown in Table 1.

Table 1: Comparison with other state of the art RTPS

\begin{tabular}{|l|l|l|l|l|}
\hline & This work & {$[3]$} & {$[4]$} & {$[5]$} \\
\hline Type & RTPS & RTPS & RTPS & RTPS \\
\hline Process & $\begin{array}{l}40 \mathrm{~nm} \text { CMOS } \\
\text { SOI }\end{array}$ & $\begin{array}{l}\text { SiGe 0.13 } \\
\text { um }\end{array}$ & $\begin{array}{l}\text { SiGe 0.13 } \\
\text { um }\end{array}$ & $\begin{array}{l}\text { BiCMOS } \\
\text { 55nm }\end{array}$ \\
\hline Topology & Differential & Differential & Differential & $\begin{array}{l}\text { Single- } \\
\text { ended }\end{array}$ \\
\hline $\begin{array}{l}\text { Frequency } \\
\text { (GHz) }\end{array}$ & $39-45 \mathrm{GHz}$ & $26.5-32.8$ & 60 & $30-50$ \\
\hline $\begin{array}{l}\text { Phase Shift } \\
(\text { degrees })\end{array}$ & 115 & $>180$ & 156 & 60 \\
\hline $\begin{array}{l}\text { Insertion Loss } \\
(\mathrm{dB})\end{array}$ & 9 & 6 & 6.2 & 5 \\
\hline $\begin{array}{l}\text { Loss Variation } \\
(\mathrm{dB})\end{array}$ & 4 & 2 & 2.2 & 2 \\
\hline Area $\left(\mathrm{mm}^{2}\right)$ & 0.214 & 0.64 & 0.33 & 0.18 \\
\hline
\end{tabular}

\section{REFERENCES}

[1] F. Voineau, B. Martineau, M. Sié, A. Ghiotto and E. Kerhervé, "A differential vertical hybrid coupler and low capacitance RF pads for millimeter-wave applications in $28 \mathrm{~nm}$ CMOS FDSOI," 2018 IEEE 18th Topical Meeting on Silicon Monolithic Integrated Circuits in RF Systems (SiRF), Anaheim, CA, 2018, pp. 57-59.

[2] T. Li and H. Wang, "A Millimeter-Wave Fully Integrated Passive Reflection-Type Phase Shifter With Transformer-Based MultiResonance Loads for $360^{\circ}$ Phase Shifting," in IEEE Transactions on Circuits and Systems I: Regular Papers, vol. 65, no. 4, pp. 1406-1419, April 2018

[3] N. Mazor, O. Katz, R. Ben-Yishay, D. Liu, A. V. Garcia and D. Elad, "SiGe based Ka-band reflection type phase shifter for integrated phased array transceivers," 2016 IEEE MTT-S International Microwave Symposium (IMS), San Francisco, CA, 2016, pp. 1-4.

[4] H. Krishnaswamy, A. Valdes-Garcia and J. Lai, "A silicon-based, allpassive, $60 \mathrm{GHz}$, 4-element, phased-array beamformer featuring a differential, reflection-type phase shifter," 2010 IEEE International Symposium on Phased Array Systems and Technology, Waltham, MA, 2010, pp. 225-232.

[5] Z. Iskandar et al., "A 30-50 GHz reflection-type phase shifter based on slow-wave coupled lines in BiCMOS $55 \mathrm{~nm}$ technology," 2016 46th European Microwave Conference (EuMC), London, 2016, pp. 14131416. 